\title{
The role of social effects in selection for animal improvement
}

\begin{abstract}
Jason B. Wolf
Faculty of Life Sciences, The University of Manchester, The Michael Smith Building, Oxford Road, Manchester M13 9PT, UK.

ABSTRACT - Quantitative genetics can be used to understand how traits will respond to artificial selection regimes, and therefore, it can be an important tool in the development of selective breeding programs for animal improvement. In order to predict the response to selection, the traditional approach in quantitative genetics partitions phenotypic variation into a heritable genetic component that contributes to the response and a non-heritable environmental component that does not. However, there is an increasing recognition that environmental variation contributed by the social environment provided by conspecifics can ultimately originate from genetically heritably traits in a population of interacting individuals, blurring the distinction between genetic and environmental variation. The presence of these 'social effects' on trait expression means the environment can provide a source of heritable variation that can contribute to trait evolution. Under some conditions, such as when there is competition, the social effects of genotypes on the phenotypes of other individuals may oppose their direct effects on their own phenotypes and, as a result, the evolution of social effects can oppose the evolution of direct genetic effects and thereby can impede the response to selection. Furthermore, when the social effects of genotypes are uncorrelated with their direct effect, the heritable variance contributed by social effects will not contribute to a response to selection on individuals, meaning that a major part of the genetic variance in a population can be unavailable to selective breeding. These constraints can be overcome by using populations of related individuals or through group selection approaches that allow for the social effects to contribute to a response to selection.
\end{abstract}

Key words: associate effects, group selection, indirect genetic effects, social effects, social environment

\section{Introduction}

Quantitative genetics has had a long history of success in predicting the response to selection in agricultural systems. However, there are a variety of traits that have been difficult to improve through selective breeding, and there are also many traits that have apparently reached limits of improvement through standard selection regimes. Many of these traits are behaviors expressed in interactions (such as aggressive behaviors) or non-behavioral traits that are influenced by the behavior of (or other traits expressed by) conspecifics individuals interact with (perhaps as a result of competitive interactions). Traits like these that are affected by the environment provided by conspecifics, or the 'social environment', differ from others sorts of traits in that they are affected by a source of environmental variation that ultimately originates from features of conspecifics (using the term 'social environment' to refer to the environment provided by conspecifics in general, whether we consider them truly 'social' species in the strict sense, whatever that may be
Costa 2006; West-Eberhard 1979). That is, the social environment experienced by an individual is attributable to traits expressed in their social partners. Because the traits that make up the social environment can be (and likely are) heritable, there is a heritable component underlying the environmental effects on trait expression. This heritable component of the environment can alter the evolutionary dynamics of traits and can, as a result, can change the types of selection regimes that are likely to be successful.

To understand how genetically based social effects on trait expression influence trait evolution it is critical to understand the standard view of phenotypic variation that underlies traditional quantitative genetic techniques. Quantitative genetics starts with the partitioning of effects on trait expression $\left(z_{f}\right.$, which is the 'phenotypic value' of trait $f$, which is the value of trait $f$ you measure for an individual) into a genetic $\left(g_{f}\right.$, which reflects all of the genetic influences contributing to trait $f$ ) and an environmental component ( $e_{f}$, the environmental deviation, which reflects all of the 
environmental contributions influencing the expression of trait $f$ :

$z_{f}=g_{f}+e_{f}$

Most often the genetic effect is the additive effect of the genotype (the additive genotypic value), which is usually referred to as the breeding value in applied quantitative genetics (see Arnold 1994). The breeding value represents the sum of the predictable effects of alleles independent of all other alleles both at the same locus and different loci in an organism, and therefore, it is what predicts the expected phenotypes of an individual's offspring (hence it is the heritable part of the genotype).

This basic model is used to partition variation in trait expression into a set of variance components. This partitioning is important for many reasons, the most significant of which is that it allows us to separate the heritable component(s) that contribute to trait evolution (the additive genetic variance) from those that do not. For example, assuming that the terms are independent from each other, we can partition variation in the expression of the trait (i.e., the phenotypic variance, $P_{f f}$ ) into additive genetic $\left(G_{f f}\right)$ and environmental (nonheritable) variances $\left(E_{f f}\right)$ :

$P_{f f}=G_{f f}+E_{f f}$

(Falconer and Mackay1996). The key is that we have switched from examining the trait value of an individual $\left(z_{f}\right)$ to describing various components of trait variation in a population of such individuals.

The additive genetic variance $\left(G_{f f}\right)$ term in equation (2) is the variance in breeding values and is presumably the only heritable component of phenotypic variation. It, therefore, translates how selection on phenotypes in one generation lead to inherited changes in phenotypes across generations. That is, by separating the heritable from the non-heritable components of variation one can understand the relative evolutionary labiality of a trait because it is the heritable variation (i.e., the additive genetic variance) that translates selection within a generation in to changes in trait means across generations (which is the evolutionary response to selection, denoted $\Delta \bar{z}_{f}$ to indicate a change in the mean phenotypic value,). One way to express the evolutionary response to selection is to view the additive variance as a measure of the (linear/additive) relationship between variation at the molecular level (allelic variation) and therefore, as a parameter that translates linear selection on phenotypes into linear selection on genotypes. The linear relationship between a trait and fitness is given by the selection gradient $\left(\beta_{f}\right)$, and together with the additive genetic variance, it can be used to predict the evolutionary response to selection:

$\Delta \bar{z}_{f}=G_{f f} \beta_{f}$

This is the so-called Breeders' Equation (Falconer and Mackay 1996), which predicts how a population will respond to a selective breeding scheme (or to natural selection), and is more usually expressed as the response to selection $(R)$ being equal to the heritability $\left(h^{2}\right)$ times the selection differential (s), which is mathematically equivalent to the expression in equation (3). Of course, the form of the Breeders' Equation given by equation (3) is the univariate case, where there is a single trait under selection and any real breeding scheme would utilize a multivariate approach, where selection acts on multiple correlated traits. However, equation (3) captures the key assumption of traditional quantitative genetics - that we can predict how traits will respond to selection by understanding the heritability of traits in terms of the proportion of phenotypic variance attributable to the breeding values of individuals.

\section{Social effects}

When social effects exist, however, there can be a component of heritable genetic variation arising from the environment provided by conspecifics. Therefore, one needs to incorporate these effects when modeling trait variation and expected responses to selection. To do so, we partition the environment into a random nonsocial component and a term attributable to some traits expressed in conspecifics:

$z_{f}=g_{f}+e_{n(f)}+S^{\prime}$

where $S^{\prime}$ is the effect of the social environment on trait expression and $e_{n(f)}$ is the sum of all other environmental effects (including non-additive genetic effects) after removing the effects attributable to social 
interactions. The ' $S$ ' is marked with a prime to indicate that the social effect is attributable to traits measured in other conspecific individuals (i.e., I will mark all terms that arise from traits of another individual with a prime throughout; see Moore et al. 1997; Wolf 2003). These social effects on trait expression have also been called 'associate effects' (Griffing 1967; 1981a) to reflect the fact that they are the effect of an individuals associates on the expression of its phenotype.

The key feature of the environment provided by conspecifics is that it reflects the contribution of another individual to trait expression in some focal individual. In most cases, social effects on trait expression arise as a result of what have been called 'interacting phenotypes' (Moore et al. 1997; Wolf et al. 1999), meaning that it is ultimately traits expressed by interacting individuals that result in affects on trait expression. Because of this, the social "environmental" effect can have a genetic basis and is, therefore, part of the genetic architecture of traits expressed in populations. Perhaps more importantly, because the social environment can have a genetic basis, it can be heritable and contribute to trait evolution. Thus, social environments can have potentially profound effects on the evolutionary dynamics of traits.

Modeling the social effect is a major problem, but one can understand the general impact of social effects by simply taking the social effect as a property of one or more individuals that a focal individual interacts with. That is, although social effects ultimately arise from traits expressed in other individuals, we can understand their importance even if we do not know (or cannot measure) the traits that contribute the social effect. This is what is known as a 'performance trait' approach (see Wolf et al. 1998), where the social effect on the phenotype of one individual is the trait of the individual(s) it interacts with (e.g., Griffing 1967; Wolf 2003; Muir 2005; Bijma 2007a,b). That is, the performance trait approach views the influence of the social partner only through its influence on the focal individual (what one might call 'social performance', which is analogous to the concept of 'maternal performance' used in models of maternal effects; Cheverud and Moore 1994).

The social effect or social performance is a trait that can be decomposed into heritable and non-heritable components as in equation (1):
$S=a_{s}+e_{s}$

Assuming that social performance is not affected by the social interaction, we can express trait $f$ in a way that includes the genetic and environmental influences on social performance. Note that terms in equation (5) are not marked with a prime because I assume that one can measure trait $S$ in any individual including the focal individual; traits are only marked with a prime when they are considered as the effect of a social partner on a focal individual. Assuming for simplicity that a pair of individuals interact, then trait $f$ can be written as:

$$
z_{f}=a_{f}+e_{f}+\left(a_{s}^{\prime}+e_{s}^{\prime}\right)
$$

where the term in parentheses is the social effect. Equation (6) shows the partitioning of genetic effects on the expression of trait $f$ into so-called 'direct' and 'indirect' genetic effects, or direct and associate effects, where, $a_{f}$, is direct genetic effect corresponding to the effect of (or mapping from) the individual's genotype on its own phenotype. The indirect genetic effect, $a_{s}^{\prime}$, corresponds to the effect of (or mapping from) the genotype of one individual on the phenotype of another individual. Such additive indirect genetic effects have also been called 'associative breeding values' (see Bijma et al. 2007a). If an individual interacts with many individuals, we may change equation (6) to reflect the fact that the expression of trait $f$ is affected by a number of social partners (n-1) in a group of $n$ individuals (Griffing 1967; Moore et al. 1997; Bijma et al. 2007a):

$$
z_{f(i)}=a_{f(i)}+e_{f(i)}+\frac{1}{n-1} \sum_{j \neq i}^{n}\left(a_{s(j)}^{\prime}+e_{s(j)}^{\prime}\right)
$$

where the additional subscript $i$ denotes the focal individuals and $j$ the social partners (e.g., $a_{s(j)}^{\prime}$ is the additive genotypic value for trait $S$ of the $j^{\text {th }}$ group member). Although I will focus on interactions between pairs of individuals to simplify the presentation of basic concepts, it's important to keep in mind that when one considers pair-wise interactions, one is looking at an extreme case where the social environment contributes to maximal amount of phenotypic variance contributed by social effects (see Bijma et al. 2007b). This suggests that looking at 
interactions between pairs of individuals may provide a more powerful approach than looking at natural scenarios where individuals interact with many social partners, but it also results in a scenario where the variation contributed by the social environment is at its maximum.

\section{Response to selection with social effects}

It is clear from equations (6) and (7) that the response of trait $f$ to selection can be, at least in part, determined by the evolution of the social effect $(S)$ since it represents a genetic/heritable source of variation in trait $f$ in a population. However, selection on individuals will only result in the evolution of the social effects when there is a genetic relationship between the phenotypic value of trait $f$ (the target of selection) and genetic variation for trait $S$. Assuming for simplicity that individuals interact in pairs we can examine the expected response to selection of trait $f$ when selection acts on trait $f$ (since I am treating the social effect as an unmeasured trait I will ignore selection acting directly on trait $S$ ). The response to selection assuming the pattern of trait expression shown in equation (6) is:

$$
\Delta \bar{z}_{f}=\beta_{f}\left(G_{f f}+G_{f s}+r\left[G_{f s}+G_{s s}\right]\right)
$$

where $G_{f s}$ is the additive genetic covariance between trait $f$ and the social effect $(S)$ - i.e., it is the covariance between individuals' breeding values for trait $f$ and the social effect on trait $f, G_{s s}$ is the additive genetic variance for the social effect and $r$ is the coefficient of relatedness. The response to selection has three terms (in the parentheses) corresponding to three different components of evolutionary response. The first term $\left(G_{f f}\right)$ corresponds to the usual response caused by changes in direct genetic effects on trait expression (as in eq. 3). The second term $\left(G_{f s}\right)$ corresponds to the change in the mean social effect caused by selection on trait $f$, where selection on one trait (trait $f$ ) results in a correlated change social performance, $S$, which then leads to an evolutionary change in trait $f$ as the mean social environment evolves (i.e., the social effect or social environment shows a correlated response to selection on trait $f)$. The third term $\left(r\left[G_{f s}+G_{s s}\right]\right)$ is the additional component of change in the mean social effect owing to interactions between relatives and arises because relatedness creates a genotype-environment correlation, where the genotypes of individuals are correlated to the social environment they experience as a result of the fact that they are related to the individuals that create the social environment. Bijma et al. (2007a, b) presents a more general case for trait evolution (based on the models of Griffing 1967, 1981a,b) where multiple individuals interact, with an ultimate goal of understanding multi-level, but the general conclusions are the same as those seen in equation (8).

Thus, equation (8) shows that selection on trait $f$ can result in an evolutionary response because the average social effect (i.e., the mean 'social environment') evolves. But the social effect only changes in response to selection on trait $f$ when there is a genetic covariance between trait $f$ and the social effect. Such a relationship may be expected in many cases, such as when there is competition, where the competitive ability of individuals affects the size of some trait $f$ and also has a social effect, via competition, on the size of trait $f$ in other individuals. In the case of competition, we assume that this relationship will be negative (i.e., $G_{f s}<0$ ), where being more competitive make an individual's value of trait $f$ larger, but because the individual takes resources from conspecifics through competitive interactions, it makes the individual's social partners smaller (see Wolf 2003). This means that the direct response to selection in equation (8) will be of opposite sign to the evolutionary change in social effects, which will therefore impede the evolutionary response to selection (Wolf 2003). This is a major challenge when it comes to social effects, where there can be an antagonistic concerted counter evolution between direct and social effects on trait expression. At the extreme, the evolutionary response to selection can actually be in the direction opposite to the selection gradient when there is a very strong negative direct-indirect genetic covariance. Furthermore, if there is no genetic relationship between direct and social effects on trait expression $\left(G_{f s}=0\right)$, then selection on trait $f$ will not lead to evolutionary changes in the social effects if interacting individuals are not related. This can also be a problem since social effects can clearly contribute a source of heritable variation for the evolution of traits, but it may be hidden in that it may not appear as part of the directly heritable 
variation available for response to individual selection (Muir 2005; Bijma et al. 2007a, b).

However, equation (8) shows that, when interactions are between relatives, then selection on individuals can result in a change in the mean social environment (i.e., a response to selection in the mean social effect) even when the direct and indirect genetic effects are not correlated (i.e., when $G_{f s}=0$ ). The response to selection would be $\Delta \bar{z}_{f}=\beta_{f} r G_{s s}$, so in effect, interactions between relatives convert the indirect (associate) genetic variance $\left(G_{s s}\right)$ into a directly heritable variance (Bijma et al. 2007a) that contributes to the response to selection of trait $f$. This highlights the importance that relatedness can play in selection regimes when there are social effects, which has lead to a number of breeding schemes that take advantage of this feature (e.g., Griffing 1981a).

\section{Response to group selection with social effects}

There are a number of approaches one can use to selection for improvement when there are social effects that exploit the social effects as a source of heritable variation, or attempt to overcome limits imposed by the antagonistic relationship between direct and social effects. For example, there has been an interest in the efficacy of group selection as a means of selecting on traits affected by the social environment (see Griffing 1967; Muir 2005; Bijma et al. 2007a, b). Group selection may be particularly important for behavioral traits and several of the best documented cases where traits showed a strong response to group selection were for behaviors, including aggression and aggression associated mortality (Muir 1996; Craig and Muir 1996), social dominance (Moore et al. 2002), and cannibalism (Wade 1976; 1977.; Muir 1996; 2005). By selecting among groups one is directly selecting on social effects whenever the mean social effects differs among groups. For example, the mean of trait $f$ (following eq. 7) in groups of two individuals would be (ignoring the environment effects):

$$
\bar{z}_{f}=\frac{1}{2}\left(a_{f(i)}+a_{f(j)}\right)+\frac{1}{2}\left(a_{s(i)}+a_{s(j)}\right)
$$

Because the breeding values for social effects contribute to the group mean, there are a number of scenarios where group selection is more efficient for producing changes in mean trait values than is ordinary individual based selection (see Bijma et al. 2007a).
These scenarios are clear from the expected response of trait $f$ to group selection:

$\Delta \overline{\mathrm{Z}}_{f}=\frac{1}{2} \beta_{f(G)}\left[G_{\mathrm{ff}}(1+r)+2 G_{f s}(1+r)+G_{\mathrm{ss}}(1+r)\right]$

where $\beta_{f(G)}$ is the selection gradient on group means. Generally, whenever individuals' phenotypes do not predict their breeding value for the social effects that they have (i.e., where $G_{f s}=0$ ) group selection will be the only way to produce a response to selection owing to social effects, assuming interactants are not. Indeed, at the extreme, when there are no direct genetic effects on trait expression (i.e., $G_{s s}=0$, making $G_{f s}=0$ ), the indirect effects contribute a component of heritable variation that can be impossible to select on in at the individual level if interactants are not related, and, clearly in these cases, selection on the group means will be more efficient (since the response to individual selection would be zero, while the response to group selection is not). Although there are a number of scenarios that can make group selection more efficient than individual selection, the most general is where indirect effects are stronger than direct effects (i.e., $G_{s s}$ $>G_{f f}$ ) (Bijma et al. 2007a). The response to group selection can be further enhanced when groups of related individuals are used since it reduces within group genetic variance and increases the among group genetic variance. Another important implication of the response to group selection shown in equation (10) is that the response will always be of the same sign as the selection gradient on group means (Muir 2005), which, as noted above, is not necessarily true of individual level selection (eq. 8).

\section{Conclusions}

Social effects are obviously often more complex than the pattern assumed in the linear equations used herein and, furthermore, pairwise interactions are probably the exception rather than the rule. However, the overall conclusions about the importance of social effects in the evolutionary response of traits to selection are mostly unaltered by these factors. Thus, it is clear that social effects can alter the expected response to individual selection, and under some conditions (such as when there is strong competition), can actually make the response to individual selection opposite to the sign of selection. The response to selection in the presence 
of social effects can be altered when interactions are between relatives because individuals are related to the social environment they experience, and under some conditions. Under some conditions, this can enhance the response to selection by making the indirect genetic variance available to selection. Finally, when social effects are stronger than direct effects, or when there is a strong negative relationship between direct and social effects, group selection approaches (where group means are used as the selection criteria rather than individual trait values) can be more efficient than individual selection approaches.

\section{Acknowledgements}

Thanks to Allen Moore for discussions during the development of this paper and to the BBSRC (UK), NERC (UK) and NSF (USA) for funding that supported part of this research.

\section{Literature Cited}

Arnold, S. J. 1994. Multivariate inheritance and evolution: A review of concepts. Pp. 17-48 in C. R. B. Boake, ed. Quantitative Genetic Studies of Behavioral Evolution. University of Chicago Press, Chicago.

Bijma, P., Muir, W. M. and J. A. M Van Arendonk. 2007a. Multilevel selection 1: Quantitative genetic of inheritance and response to selection. Genetics 175:277-288

Bijma, P., Muir, W. M., E. D. Ellen, J. B. Wolf and J. A. M Van Arendonk. 2007b. Multilevel selection 2: Estimating the genetic parameters determining inheritance and response to selection. Genetics 175:289-299

Cheverud, J. M. and A. J. Moore. 1994. Quantitative genetic and the role of the environment provided by relatives in behavioral evolution. pages 67-100 in C. R. B. Boake ed. Quantitative Genetic Studies of Behavioral Evolution. University of Chicago Press.
Costa, J. T. 2006. The Other Insect Societies. Harvard University Press, Cambridge, MA.

Craig, J. V., and W. M. Muir, 1996 Group selection for adaptation to multiple-hen cages: beak-related mortality, feathering and body weight responses. Poult. Sci. 75: 294302.

Falconer, D. D., and T. F. C. Mackay. 1996. Introduction to quantitative genetics, 4th edition. Longman, Essex.

Griffing, B., 1967 Selection in reference to biological groups. I. Individual and group selection applied to populations of unordered groups. Aust. J. Biol. Sci. 20: 127.

Griffing, B., 1981a A theory of natural-selection incorporating interaction among individuals. I. The modeling process. J. Theor. Biol. 89: 635-658.

Griffing, B., 1981b A theory of natural-selection incorporating interaction among individuals. II. Use of related groups. J. Theor. Biol. 89: 659-677.

Moore, A. J., E. D. Brodie, III, and J. B. Wolf. 1997. Interacting phenotypes and the evolutionary process: I. direct and indirect genetic effects of social interactions. Evolution 51:1352-1362.

Moore, A. J., K. F. Haynes, R. F. Preziosi \& P. J. Moore. 2002. The evolution of interacting phenotypes: genetics and evolution of social dominance. American Naturalist 160: S186-S197.

Muir, W. M., 1996 Group selection for adaptation to multiplehen cages: selection programand direct responses. Poult. Sci. 75: 447-458.

Muir,W. M., 2005 Incorporation of competitive effects in forest tree or animal breeding programs. Genetics 170: 1247-1259.

Wade, M. J., 1976 Group selection among laboratory populations of Tribolium. Proc. Natl. Acad. Sci. USA 73: 4604-4607.

Wade, M. J., 1977 An experimental study of group selection. Evolution 31: 134-153.

West-Eberhard, M. J. 1979. Sexual selection, social competition and evolution. Proceedings of the American Philosophical Society 123:222-234.

Wolf, J. B. 2003. Genetic architecture and evolutionary constraint when the environment contains genes. Proceedings of the National Academy of Sciences of the United States of America 100:4655-4660.

Wolf, J. B., E. D. Brodie III, J. M. Cheverud, A. J. Moore and M. J. Wade. 1998. Evolutionary consequences of indirect genetic effects. Trends in Ecology and Evolution 13:64-69. 